\title{
Lose biodiversity, gain disease
}

Hamish Ian McCallum ${ }^{1}$

Environmental Futures Research Institute, Griffith University, Brisbane, QLD 4111, Australia

There has been a vigorous and sometimes acerbic debate about the generality of the "dilution effect": the notion that biodiversity inhibits infectious disease, and conversely that loss of biodiversity increases disease risks to humans and livestock. In PNAS, Civitello et al. (1) report a meta-analysis of more than 200 individual effect sizes for 61 parasite species, and find strong support for the dilution effect. One of the key utilitarian arguments for preserving biodiversity is that it provides "ecosystem services," services that are essential for human survival and well-being $(2,3)$. If the dilution effect is indeed a general phenomenon, it would be an important ecosystem service, with wide public policy implications.

The hypothesis that biological diversity limits infectious disease goes back to at least the middle of last century. In his classic paper on the epidemiology of malaria, MacDonald (4) suggested controlling malaria through "zooprophylaxis," the provision of alternative hosts (such as cattle) that mosquito vectors prefer to bite over humans, thereby limiting transmission to humans. In a similar but more general vein, Elton (5) suggested that simple communities could be invaded more easily than complex communities. In an influential body of work, summarized in ref. 6, Ostfeld and Keesing have trenchantly argued that biodiversity is an effective buffer against infectious disease threats.

\section{Lyme Disease}

Much of Ostfeld and Keesing's (6, 7) work is based on a single, albeit important case study: Lyme disease in the northeast of the United States. Lyme disease in humans is a debilitating illness caused by the spirochete Borrelia burgdorferi, which is transmitted to humans via ticks, primarily the nymphal stage of Ixodes spp. (7). The nymphal ticks are host generalists, feeding on a variety of mammal species. One, the white-footed mouse Peromyscus leucopus, is a particularly competent host for the spirochete and as a smallbodied habitat generalist, persists in the smallest habitat patches (7). Where a high diversity of alternative hosts is present, many ticks will feed on these species, most of which are less-competent reservoirs for Borrelia, reducing the likelihood that nymphal ticks will transmit the infection to humans. Whether this conclusion can be generalized across the range of Lyme disease in North America and whether it applies at broader spatial scales is debated $(8,9)$. A recent study of the fleavectored zoonotic pathogen Bartonella in Africa found increased prevalence of the pathogen in rodents as large wildlife declined (10). This result shows that increased risks to humans from pathogens with rodent reservoir hosts following loss of biodiversity are not restricted to Lyme disease.

Whatever might be the case with Lyme disease and Bartonella, several authors dispute

\section{The meta-analysis of Civitello et al. is particu- larly important because it draws together as many studies as possible of the dilution effect.}

the extent to which a dilution effect is a general phenomenon. These authors argue that changes in disease risk with loss of biodiversity, from dilution to amplification, are idiosyncratic responses to the specifics of individual species and host-parasite communities $(11,12)$. In this context, the metaanalysis of Civitello et al. (1) is particularly important because it draws together as many studies as possible of the dilution effect. It is not the first such study; an earlier metaanalysis concluded that there was no general effect of biodiversity on disease, supporting the idiosyncratic response hypothesis (13). That study was, however, based on only 16 case studies of 6 zoonotic pathogens. Although not a formal meta-analysis, Wood et al. (11) compiled a list of the 69 most common pathogenic metazoan and protozoan parasites of humans, and suggested biodiversity may reduce human disease in only $12 \%$ of these, whereas it may increase disease in $38 \%$. In the remaining cases, the relationship was either neutral or indeterminable. In Wood et al.'s study, the nature of the relationship between biodiversity and disease impact was inferred, rather than directly measured, and the case studies did not include viruses or bacteria.

\section{Loss of Biodiversity, Not Biodiversity} per se, Affects Disease

Clearly the hypothesis should not be simply that biodiversity reduces disease threats. There are more human pathogens and parasites in the tropics than in temperate regions (14), and tropical regions are more biodiverse. One would also expect that a higher diversity of wild potential host species should lead to a higher number of pathogens available to infect humans and livestock. There are numerous examples in which human populations close to biodiverse environments, such as forests, are more severely affected by pathogens than are populations further from biodiverse environments (11). The dilution effect hypothesis instead proposes that, given an existing community of hosts and parasites, deletion of some of the potential host species is likely to increase parasite or pathogen burdens in the remaining species, including humans.

An important question here is whether there is a systematic relationship between the order in which species are lost from ecosystems and their competence as hosts for pathogens. Habitat destruction and other forms of disturbance do not randomly delete species from local communities. A negative association between extirpation risk and host competence would provide a possible general mechanism for the dilution effect. Several studies have found such an association. In vertebrates, the largest-bodied and longestlived species are often extirpated first. These species often invest more in immunological defenses than coexisting smaller-bodied species (15). Frog species that are competent hosts for the virulent trematode pathogen Ribeiroia ondatrae inhabit low-diversity Californian ponds, whereas less-competent frogs are also present in higher-diversity ponds (16). Ubiquitous plant species are the most competent hosts of both aphids and aphid-vectored plant pathogens, leading to higher prevalence in species-poor plant communities (17).

Author contributions: H.I.M. wrote the paper

The author declares no conflict of interest.

See companion article on page 8667

${ }^{1}$ Email: h.mccallum@griffith.edu.au. 
Inevitably, this meta-analysis (1) has a number of limitations. First, a majority of the studies analyzed compared pathogen burden in the presence of one host species with pathogen burden when one additional host species was added. Many people would not consider this a dilution effect. Nevertheless, meta-analysis of 44 empirical studies spanning a gradient of diversity found a dilution effect, suggesting that the results can be generalized beyond single versus two hostspecies situations. Second, most of the individual case studies identified a "focal host" and investigated how its level of infectious disease depended on the presence or diversity of other hosts. In the case of zoonotic pathogens, humans are the obvious focal host. For pathogens of nonhuman animals and plant pathogens, selecting focal hosts is more arbitrary. Third, metaanalyses can only report on published papers. A common criticism is that of publication bias: significant results are more likely to be published (18). A more substantial issue here (1) is how additional species were selected by the generators of the primary research. There may have been bias toward adding species that were partially resistant to the pathogen rather than adding highly competent species.

Nevertheless, the results (1) are highly statistically significant, no matter how the studies are subdivided, providing confidence that the conclusions are general and robust. Dilution effects are apparent for both human and wildlife parasites, for parasites with simple and complex life histories, for microparasites and macroparasites, for specialist and generalist parasites, and in both manipulative and observational studies. Similar patterns were also observed in a parallel analysis of the effect of plant biodiversity on the impact of herbivores, suggesting that the dilution effect may be broadly applicable to natural enemies.

\section{Where to Next?}

Civitello et al.'s study (1) is unlikely to be the last word on this important topic. As with most good pieces of research, although it answers some questions, it raises many others, providing clear directions for future work. In particular, the authors call for more

1 Civitello DJ, et al. (2015) Biodiversity inhibits parasites: Broad evidence for the dilution effect. Proc Natl Acad Sci USA 112:8667-8671.

2 Ehrlich PR, Mooney HA (1983) Extinction, substitution, and ecosystem services. Bioscience 33(4):248-254.

3 Myers N (1996) Environmental services of biodiversity. Proc Nat Acad Sci USA 93(7):2764-2769

4 MacDonald G (1957) The Epidemiology and Control of Malaria (Oxford Univ Press, London).

5 Elton CS (1958) The Ecology of Invasions by Animals and Plants (Methuen, London).

6 Ostfeld RS, Keesing F (2012) Effects of host diversity on infectious disease. Annu Rev Ecol Evol Syst 43:157-182.

7 Ostfeld RS, Keesing F (2000) Biodiversity and disease risk: The case of Lyme disease. Conserv Biol 14(3):722-728.

8 Wood CL, Lafferty KD (2013) Biodiversity and disease: A synthesis of ecological perspectives on Lyme disease transmission. Trends Ecol Evol 28(4):239-247

9 Ostfeld RS, Keesing F (2013) Straw men don't get Lyme disease: Response to Wood and Lafferty. Trends Ecol Evol 28(9):502-503. 10 Young HS, et al. (2014) Declines in large wildlife increase landscape-level prevalence of rodent-borne disease in Africa. Proc Natl Acad Sci USA 111(19):7036-7041. ecologically relevant experimental tests of the dilution effect over a broader range of host diversity. They also identify the need to move beyond the increasingly polarized debate about the generality of dilution and toward a mechanistic framework capable of predicting when biodiversity will amplify or dilute infectious disease. Civitello et al. provide a quantitative and systematic analysis of a broad range of experimental and observational studies, rather than a list cherry-picked to support a predetermined conclusion. By doing so they have substantially advanced our understanding of the relationship between biodiversity and disease risk, providing a platform for future research.

11 Wood CL, et al. (2014) Does biodiversity protect humans against infectious disease? Ecology 95(4):817-832.

12 Randolph SE, Dobson ADM (2012) Pangloss revisited: A critique of the dilution effect and the biodiversity-buffers-disease paradigm. Parasitology 139(7):847-863.

13 Salkeld DJ, Padgett KA, Jones JH (2013) A meta-analysis suggesting that the relationship between biodiversity and risk of zoonotic pathogen transmission is idiosyncratic. Ecol Lett 16(5): 679-686.

14 Guernier V, Hochberg ME, Guégan J-F (2004) Ecology drives the worldwide distribution of human diseases. PLOS Biol 2(6):e141.

15 Joseph MB, Mihaljevic JR, Orlofske SA, Paull SH (2013) Does life history mediate changing disease risk when communities disassemble? Ecol Lett 16(11):1405-1412.

16 Johnson PTJ, Preston DL, Hoverman JT, Richgels KLD (2013) Biodiversity decreases disease through predictable changes in host community competence. Nature 494(7436):230-233.

17 Lacroix C, et al. (2014) Non-random biodiversity loss underlies predictable increases in viral disease prevalence. $J R$ Soc Interface 11(92):20130947.

18 Rothstein HR, Sutton AJ, Borenstein M (2006) Publication Bias in Meta-Analysis (John Wiley \& Sons, Chichester, UK). 